\title{
Modelling High-Frequency Volatility with Three-State FIGARCH models
}

\author{
$\underline{\text { Y.Shi }}^{\text {a }}{ }^{\text {K.Y-. Ho }}{ }^{\text {a }}$ \\ ${ }^{a}$ Research School of Finance, Actuarial Studies and Applied Statistics, The Australian National University, \\ Canberra, Australia \\ Email: yanlin.shi@anu.edu.au
}

\begin{abstract}
Fractionally Integrated Generalized Autoregressive Conditional Heteroskedasticity (FIGARCH) models have enjoyed considerable popularity over the past decade because of its ability to capture the volatility clustering and estimate long memory of conditional volatility. However, when structural breaks are present, it is well known that the estimate of long memory will be spurious. Consequently, two approaches are developed to incorporate the structural breaks into FIGARCH framework. First, the intercept in the conditional variance equation is modelled via a certain function of time. The Adaptive-FIGARCH (A-FIGARCH) and Time-Varying FIGARCH (TV-FIGARCH) models are proposed based on this idea. Second, financial series are modelled in separate stages. At the first stage, certain algorithm is applied to detect the change points. FIGARCH model is then fitted, with the intercept (and other parameters) being allowed to vary between change points. A recently developed such algorithm Nonparametric Change Point Model (NPCPM) can be extended to the FIGARCH framework, which is the NPCPM-FIGARCH model. We adopt the second approach but use Markov Regime-Switching (MRS) model to detect the change points and identify three economic states depending on the scale of volatility. This new 2-stage Three-State FIGARCH (3S-FIGARCH) framework and other FIGARCH models are fitted to the hourly data set composed of four major stock indexes, with Gaussian and non-Gaussian distribution assumptions, individually. From the comparison, we find that our model can potentially give an improved fit with better estimate of long memory parameter.
\end{abstract}

Keywords: Long Memory, Structural Breaks, FIGARCH, Change Detection 


\section{INTRODUCTION}

Long memory persistence describes the property of financial series, whose sample autocorrelations are significantly different from zero even for large lags (Baillie et al., 1996). Although it has different definitions, that used by Diebold and Inoue (2001) is widely accepted as $\operatorname{var}\left(S_{T}\right)=O\left(T^{2 d+1}\right)$, where $S_{T}=\sum_{t=1}^{T} y_{t},\left\{y_{t}\right\}$ is a sequence of interested financial series and $T$ is the number of observations. Then $d$ is the long memory parameter, and $d>0$ indicates the existence of long memory.

Particularly, time-varying volatility of financial return has been a considerable field of research, ever since the introduction of the univariate generalized Autoregressive Conditional Heteroskedasticity (GARCH) model by Bollerslev (1986). The extension of this idea to model the long memory persistence leads to the outcome of Fractionally Integrated GARCH (FIGARCH) model, which is based on the application of the fractional differencing operator to the autoregressive structure of the conditional variance by assuming that it follows a hyperbolic rather than exponential decay (Baillie et al., 1996). FIGARCH model has thus received considerable interest because of its ability to capture the persistence in the volatility (Ho et al., 2013). However, same as the main weakness of the original GARCH model, it assumes that the conditional volatility has only one regime over the entire period. Unfortunately, it is not always true. To overcome this drawback, many studies have suggested that structural breaks should be combined into the long memory models to properly fit financial return volatility (Baillie and Morana, 2009; Belkhouja and Boutahary, 2011). Adaptive-FIGARCH (A-FIGARCH) model proposed by Baillie and Morana (2009) and Time-Varying FIGARCH (TV-FIGARCH) model proposed by Belkhouja and Boutahary (2011) are developed based on this idea, by modelling the intercept in the conditional variance equation via certain function of time.

Among the existing literatures, another approach to incorporate the structural breaks in GARCH frame work is to fit the model in stages (Ross, 2013). First, the return series is fitted by certain algorithm to detect the abrupt change points. Then, intercept in the conditional variance equation is allowed to be different for each period between the change points. Traditionally, iterated cumulative sum of squares (ICSS) algorithm is employed to discover the change points. However, as pointed out by Ross (2013), ICSS only works for financial series following a Gaussian distribution. To overcome this drawback, Ross (2013) develops the Nonparametric Change Point Model (NPCPM) algorithm, which employs the Mood test to detect the change points and proposes the NPCPM-GARCH model. This idea can be straightforwardly extended to the FIGARCH model to incorporate the structural breaks in the long memory framework.

A potential problem of the NPCPM and ICSS algorithms is that they identify the change points without considering the economic states. For example, sample periods with different structures are detected based on the change points only, but will not be combined and studied later by their economic similarity. Therefore, it may lead to a non-parsimonious model because of the negligence of similarity. In addition, the volatility series modelled in this way is assumed to switch permanently to different regimes, rather than have a probability to switch back to the same regime later. Besides, since NPCPM and ICSS both require the return series to be independent, Ross (2013) suggests that NPCPM should be applied to the standardized residuals from (FI)GARCH model. However, in the GARCH framework, standardized residuals are assumed to be independently and identically distributed. As a result, it can cause some problem by detecting change points based on them, such as interpretation of the detected change points.

In this paper, we propose a two-stage Three-State FIGARCH (3S-FIGARCH) model which also incorporates the structural breaks by modelling the FIGARCH process in stages. At the first stage, Markov RegimeSwitching (MRS) framework proposed by Hamilton (1989) is employed to detect change points directly. The MRS model assumes that there are two economic states (low- and high-volatility states) of the financial series, and the series can switch between them along time, with the state at time $t$ being a stationary, irreducible Markov process. In this study, we fit the MRS model with innovations assumed to follow a Student-t distribution to estimate the smoothing probability series of low-volatility state. We further use the classification of economic states argued by Wilfling (2009), where there are three states: "calm" (extremely low-volatility), "turbulent" (extremely high-volatility) and "intermediate" (the others) states. Furthermore, we set two threshold probabilities. Then, at time $t$, return series is assumed to lie in "calm" state when the smoothing probability is less than the lower threshold, in "turbulent" state when it is greater than the upper threshold and in "intermediate" state otherwise. At the second stage, intercept in conditional variance equation of FIGARCH process is allowed to be different for each state. Since there are only three possible values the intercept, our model should be more parsimonious than NPCPM framework. Moreover, MRS model does not require the financial series to be originally independent, so that the detected states (change points) are more reliable. Finally, MRS 
model takes the economic information (low and high volatility) of the return series into consideration, so that the detected states can be interpreted economically.

To empirically compare the 3S-FIGARCH model with original FIGARCH and existing time-varying FIGARCH frameworks including A-FIGARCH, TV-FIGARCH and NPCPM-FIGARCH models, we apply them to two world stock indexes. They are hourly data including (1) the NASDAQ Composite (Nasdaq) which consists of over 3000 stocks listed on the NASDAQ stock Market, and (2) Australian Securities Exchange 50 (ASX) which consists of 50 large Australian companies. Assumptions of Gaussian and Student-t distributions are modelled individually for each model and stock index. ${ }^{1}$ The results suggest that our method generally gives a better fit to the data when measured using standard criteria and gives a betterd estimate long memory parameter. Also, models with Student-t distribution assumption overall outperform models with Gaussian distribution assumption. Thus, our 3S-FIGARCH framework could be a widely useful tool for modelling long memory persistence of high-frequency financial volatility in other contexts.

\section{The Original and Existing Time-Varying FigarCH Models}

\subsection{The original FIGARCH Model}

FIGARCH model is proposed by Baillie et al. (1996), which is extended from GARCH family models. As concluded by Ross (2013), GARCH-family models have enjoyed popularity among academics because of their ability to capture some of the typical stylized facts of financial time series, such as volatility clustering. Franses and van Dijk (1996) show that GARCH-family models take into account the feature of time-varying volatility over a long period of time and provide good in-sample estimate. Apart from the above features, FIGARCH model is particularly designed to model the long memory characteristic.

The original FIGARCH( $1, d, 1)$ model is described as follows.

$$
r_{t}=\mu+\varepsilon_{t}, \varepsilon_{t}=\eta_{t} \sqrt{h_{t}} \text { and }\left(1-b_{1} L\right) h_{t}=\omega+\left[\left(1-b_{1} L\right)-\left(1-\varphi_{1} L\right)(1-L)^{d}\right] \varepsilon_{t}^{2}
$$

where $\varepsilon_{t}$ is the error at time $t$. $h_{t}$ is the conditional volatility of $\varepsilon_{t}$ at time $t . \eta_{t}$ is an identical and independent sequence following a specific distribution. $L$ is the lag operator. $(1-L)^{d}$ is the fractional differencing operator. $d$ is the long memory parameter. We have a stationary long memory process when $0<d<1$. If $d=1$, the process has a unit root and thus a permanent shock effect, which is equivalent as the IGARCH model. If $d=0$, the process reduces to an ordinary GARCH process without long memory property (Baillie et al., 1996).

\subsection{A-FIGARCH Model}

The main weakness of the original FIGARCH model is that it assumes that the conditional volatility has only one regime over the entire period, which can lead to spurious regression. In order to overcome such drawback, Baillie and Morana (2009) points out that a powerful approach is to allow the intercept to be time dependent. Compared with the FIGARCH model, only the conditional equation is changed, which is described as follows.

$$
b(L) h_{t}=\omega+\left[b(L)-\varphi(L)(1-L)^{d}\right] \varepsilon_{t}^{2}+\omega_{t} \text { and } \omega_{t}=\sum_{j=1}^{k}\left[\gamma_{j} \sin (2 \pi j t / T)+\delta_{j} \cos (2 \pi j t / T)\right]
$$

where $T$ is the number of observations. Moreover, they argue that adequate approximations can be achieved with very parsimonious specifications of only $k=1$ or 2 .

\subsection{TV-FIGARCH Model}

Belkhouja and Boutahary (2011) provide another approach to model the variation in the intercept and propose the TV-FIGARCH model. The conditional equation of their model is constructed as follows.

$$
\begin{gathered}
b(L) h_{t}=\omega+\left[b(L)-\varphi(L)(1-L)^{d}\right] \varepsilon_{t}^{2}+\sum_{r=1}^{R} \omega_{r} F_{r}\left(s_{t}, \gamma_{r}, c_{r}\right) \\
\text { where } s_{t}=t / T \text { and } F_{r}\left(s_{t}, \gamma_{r}, c_{r}\right)=\left(1+\exp \left\{-\gamma_{r}\left(s_{t}-c_{r}\right)\right\}\right)^{-1}
\end{gathered}
$$

In addition, $\gamma_{r}$ controls the degree of smoothness and must be positive, while $c_{r}$ is the threshold parameter with constrain $c_{1} \leq c_{2} \leq \ldots \leq c_{R} . s_{t}=t / T$ is the transition variable. When $\gamma_{r} \rightarrow \infty$, the switch from one state to another is abrupt, that is, a smooth change approaches a structural break at the threshold parameter $c_{r}$.

\footnotetext{
${ }^{1}$ The reason to use Student-t distribution is that in real economy, financial series is rarely Gaussian but typically leptokurtic and exhibits heavy-tail behaviour (Susmel and Engle, 1994). Therefore, distributions which can capture those properties will be expected to lead to more efficient estimation. Since Student-t is a widely used alternative in finance study (Ho et al., 2013), it is employed in this paper to be compared with Gaussian distribution.
} 


\subsection{NPCPM-FIGARCH Model}

Another approach to incorporate the structural breaks is to fit the GARCH family models in stages (Ross, 2013). We extend the idea of NPCPM-GARCH model to the FIGARCH model, and the conditional equation of NPCPM-FIGARCH model is:

$$
b(L) h_{t}=\omega_{t}+\left[b(L)-\varphi(L)(1-L)^{d}\right] \varepsilon_{t}^{2}
$$

where $\omega_{t}$ is equal to some constant $k_{0}$ until the first change point, then switches to $k_{1}$ until the next change point, and so on.

\section{THE 3S-FIGARCH MODEL}

\subsection{Step 1: Markov Regime-Switching Model with Student-t Innovation (MRS-t)}

As discussed in the introduction, a potential problem of existing algorithms to detect change point is that they fail to take economic information into consideration. Therefore, we propose a new approach, which employs MRS model to identify the economic states of financial series.

MRS model is proposed by Hamilton (1989). Let $\left\{s_{t}\right\}$ be a stationary, irreducible Markov process with discrete state space $\{1,2\}$ and transition matrix $P=\left[p_{j k}\right]$ where $p_{j k}=\mathrm{P}\left(s_{t+1}=k \mid s_{t}=j\right)$ is the transition probability of moving from state $j$ to state $k(j, k \in\{1,2\})$. Then, we have a MRS-t model:

$$
r_{t}=\mu+\varepsilon_{s_{t}, t} \text { and } \varepsilon_{s_{t}, t}=\sigma_{s_{t}} \eta_{t} \text { where } \eta_{t} \stackrel{i i d}{\sim} t(0,1, v)
$$

$\varepsilon_{s_{t}, t}$ is the error at time $t$ in state $s_{t} . \eta_{t}$ is an identical and independent sequence following Student-t distribution, with 0 mean and unit standard deviation. $v$ is the degree of freedom of the Student-t distribution ${ }^{2} . \sigma_{s_{t}}$ is the standard deviation of $\varepsilon_{s_{t}, t}$ at time $t$ in state $s_{t}$. In this paper, we constrain that $\sigma_{1}<\sigma_{2}$ so that state 1 and 2 will indicate the "calm" (low-volatility) and "turbulent" (high-volatility) economic states respectively for the interested financial series. The parameters of the MRS-t model are estimated using the maximum likelihood estimation (MLE) described in Hamilton (1989).

In order to identify which economic state the financial series lies in at time $t$, we extract the smoothing probability of "calm" state as follows (Hamilton, 1989).

$$
P\left(s_{t}=1 \mid \theta, \Omega_{T}\right)=\omega_{1, t}\left[\frac{p_{11} P\left(s_{t+1}=1 \mid \theta, \Omega_{T}\right)}{P\left(s_{t+1}=1 \mid \theta, \Omega_{t}\right)}+\frac{p_{12} P\left(s_{t+1}=2 \mid \theta, \Omega_{T}\right)}{P\left(s_{t+1}=2 \mid \theta, \Omega_{t}\right)}\right],
$$

where $\Omega_{t-1}$ is the information set at time $t-1 . \theta$ is the vector of parameters. $\omega_{j, t-1}$ is the filtered probability in state $j$ at time $t-1$, which is equal to $P\left(s_{t-1}=j \mid \theta, \Omega_{t-1}\right)^{3}$. Using the fact that $P\left(s_{T}=1 \mid \theta, \Omega_{T}\right)=\omega_{1, T}$, the smoothing probability series $P\left(s_{t}=1 \mid \theta, \Omega_{T}\right)$ can be generated by iterating (6) backward from $T$ to 1 .

As suggested by Hamilton (1989), a widely recognized rule is that if $P\left(s_{t}=1 \mid \theta, \Omega_{T}\right)$ less than $0.5, r_{t}$ is assumed to lie in the "calm" state and otherwise in the "turbulent" state. In addition, as argued by Wilfling (2009), $r_{t}$ lies in extremely high-volatility state when $P\left(s_{t}=1 \mid \theta, \Omega_{T}\right)$ is close to 0 , in extremely low-volatility state when $P\left(s_{t}=1 \mid \theta, \Omega_{T}\right)$ is close to 1 and in intermediate state otherwise. Following this idea, we further redefine three economic states. If $P\left(s_{t}=1 \mid \theta, \Omega_{T}\right)<P_{1}, r_{t}$ lies in the "turbulent" state. If $P\left(s_{t}=1 \mid \theta, \Omega_{T}\right)>$ $P_{2}, r_{t}$ lies in the "calm" state. Otherwise, $r_{t}$ lies in the "intermediate" state.

\subsection{Step 2: FIGARCH Modelling}

After identifying the three economic states, we can fit the 3S-FIGARCH model with conditional variance equation as follows.

$$
b(L) h_{t}=\omega_{s_{t}}+\left[b(L)-\varphi(L)(1-L)^{d}\right] \varepsilon_{t}^{2}
$$

where $\omega_{s_{t}}$ is the intercept at time $t$ in state $s_{t}$. Since there are only three economic states in this framework, $\omega_{s_{t}}$ will have three possible values, which satisfy the condition $\omega_{1}<\omega_{2}<\omega_{3}(1,2$ and 3 indicate the "calm", "intermediate" and "turbulent" states, respectively).

\footnotetext{
${ }^{2}$ As noted by Haas and Paolella (2012), if regimes are not Gaussian but leptokurtic, the use of within-regime normality can seriously affect the identification of the regime process. As to the reason, they argue that Quasi MLE (QMLE) based on Gaussian components does not provide a consistent estimator of MRS model. Therefore, we use Studnet-t distribution instead of the Gaussian distribution ${ }^{3}$ See Hamilton (1989) for how to estimate the filtered probability.
} 


\section{EMPIRICAL RESULTS}

We apply FIGARCH models to two world stock indexes as described in section 1, the Nasdaq and ASX. The hourly closing prices for each index over the period 1/1/2001 to 31/12/2012 are obtained from Thomson Reuters Tick History (TRTH) database.

For each index, the return series in percentage is defined as the logarithm of the hourly closing price differences times 100, that is, $r_{t}=100 \times \log \left(S_{t} / S_{t-1}\right)$, where $S_{t}$ is the hourly closing price at time $t$. For the descriptive statistics ${ }^{4}$, the means of both stock returns are close to 0 . Standard deviations are around 0.5 and 0.4 for Nasdaq and ASX, respectively. For kurtosis, neither of them are close to 0, indicating a non-Gaussian distribution. To further confirm it, p-values from both Kolmogorov-Smirnov and Jarque-Bera normality tests are close to 0 , suggesting rejection of null hypothesis that return is normally distributed. Finally, Ljung-Box test indicates that both stock indexes have significant autocorrelation in the squared return, suggesting a time-varying volatility.
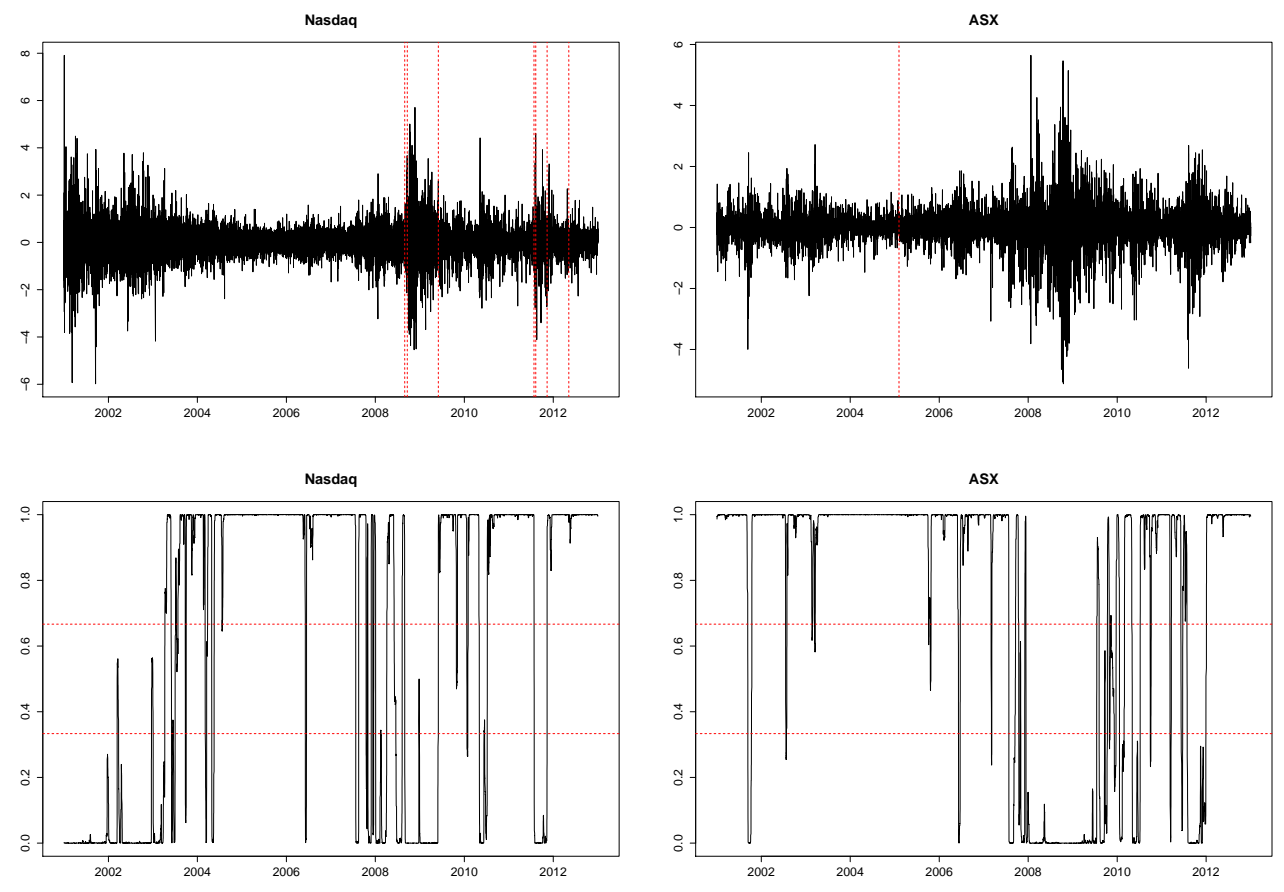

Figure 1. Hourly Closing Prices for the Stock Indexes

\subsection{Detected Change Points}

To fit the NPCPM-FIGARCH and 3S-FIGARCH models, we need to firstly detect the change points. As noted by Ross (2013), Mood test only works for independent series. Therefore, we also fit original FIGARCH model for the stock indexes to extract the conditional variance series $h_{t}$. Then, we apply the NPCPM algorithm to the standardized residuals $\varepsilon_{t} / \sqrt{h_{t}}$ to detect the change points ${ }^{5}$. The discovered change points, along with return series of both indexes are plotted in Figure 1. There are 7 and 1 change point(s) found for Nasdaq and ASX,respectively.

As described in section 3.1, MRS-t model is fitted for both indexes to extract $P\left(s_{t}=1 \mid \theta, \Omega_{T}\right)$ series. Without loss of generality, we set the probability to lie in any of the three economic states the same by letting $P_{1}=1 / 3$ and $P_{2}=2 / 3$. Then, smoothing probability series $P\left(s_{t}=1 \mid \theta, \Omega_{T}\right)$ along with threshold probabilities $P_{1}$ and $P_{2}$ are plotted in Figure 1. Generally speaking, both indexes lie in the "turbulent" state in the 2008 Global Financial Crisis (GFC) period. From 2001 to 2003, Nasdaq stays in the "turbulent" state, which could be due

\footnotetext{
${ }^{4}$ They are not presented in this paper and are available upon request

${ }^{5}$ We use cpm package in R to apply the NPCPM algorithm. Particularly, to minimize the false identified change points, ARL0 parameter in cpm is set to 50000. We thank Ross to make the package available.
} 
to the effect of IT bubble cracks at the beginning of 21 century. It seems that Australian stocks are not affected seriously, and ASX stays in "calm" state during that period. Between 2003 and 2008, both of them tend to lie in the "calm" state. After 2010 (end of 2008 GFC), both indexes seem to switch back to and stay in "calm" state in most of the time. In conclusion, the identification of economic states for both indexes is consistent with the real macroeconomic situation.

Table 1. Summary Outputs of Various FIGARCH Models for Stock Indexes

\begin{tabular}{|c|c|c|c|c|c|c|c|c|}
\hline & \multicolumn{4}{|l|}{ Nasdaq } & \multicolumn{4}{|l|}{ ASX } \\
\hline & $d$ & log.lik & $A I C$ & $B I C$ & $d$ & $\log . \operatorname{lik}$ & $A I C$ & $B I C$ \\
\hline \multicolumn{9}{|c|}{ Panel A: Normal Distribution } \\
\hline FIGARCH & 0.3841 & -13823 & 27657 & 27697 & 0.3021 & -5969 & 11948 & 11988 \\
\hline A-FIGARCH & 0.3843 & -13816 & 27645 & 27701 & 0.2740 & -5906 & 11825 & 11881 \\
\hline TV-FIGARCH & 0.3509 & -13788 & 27592 & 27655 & 0.2848 & -5917 & 11851 & 11915 \\
\hline NPCPM-FIGARCH & 0.3545 & -13777 & 27578 & 27674 & 0.2882 & -5938 & 11888 & 11936 \\
\hline 3S-FIGARCH & 0.2162 & -13623 & 27259 & 27315 & 0.1600 & -5662 & 11338 & 11394 \\
\hline \multicolumn{9}{|c|}{ Panel B: Student-t Distribution } \\
\hline FIGARCH & 0.5374 & -11456 & 22924 & 22971 & 0.3695 & -880 & 1772 & 1819 \\
\hline A-FIGARCH & 0.5130 & -11446 & 22907 & 22971 & 0.3982 & -828 & 1672 & 1735 \\
\hline TV-FIGARCH & 0.5078 & -11434 & 22885 & 22957 & 0.3764 & -841 & 1701 & 1773 \\
\hline NPCPM-FIGARCH & 0.4581 & -11387 & 22800 & 22903 & 0.3701 & -842 & 1697 & 1753 \\
\hline 3S-FIGARCH & 0.3458 & -11257 & 22529 & 22593 & 0.3361 & -753 & 1522 & 1586 \\
\hline
\end{tabular}

\subsection{Model Performance Comparison}

After detecting the change points for all the stock indexes, NPCPM-FIGARCH and 3S-FIGARCH ${ }^{6}$ models can be fitted, along with the original FIGARCH, A-FIGARCH and TV-FIGARCH models ${ }^{7}$. All models are fitted with $(1, d, 1)$ specification. The logarithm of likelihood (log.lik), Akaike Information Criterion (AIC) and Bayesian Information Criterion (BIC) under different distribution of innovation assumptions are presented in Table 1. In addition, as argued by Diebold and Inoue (2001), long memory can be caused by structural breaks and is easily confused with it. Therefore, we will expect that if structural breaks are present and are correctly modelled, the estimate of long memory parameter will be smaller. As a result, estimates of $d$ are also reported in Table 1 for comparison.

For the original FIGARCH models, those using Student-t distribution outperform the Gaussian models for both the stock indexes. This result is consistent with the fact that Gaussian distribution assumptions are rejected in both cases. Turning to the long memory parameter $d$, almost all the estimates are less than 0.5 , suggesting that the two stock indexes have stationary conditional volatility from 1/1/2001 to 31/12/2012.

In terms of A-FIGARCH, TV-FIGARCH and NPCPM-FIGARCH models, there are some common conclusions for both indexes. First, by comparing AIC and BIC, all three models overall outperform the original FIGARCH models. This suggests that incorporating the structural breaks can lead to better model performance. More specifically, TV-FIGARCH models generally outperform A-FIGARCH models for Nasdaq. For ASX, A-FIGARCH models have smaller AIC and BIC than TV-FIGARCH models. More importantly, NPCPMFIGARCH models outperform both A-FIGARCH and TV-FIGARCH models in all cases. This indicates that allowing intercept to vary according to the detected change points has better performance than modelling it via time dependent functions. As to the estimates of $d$, A-FIGARCH, TV-FIGARCH and NPCPM-FIGARCH models tend to generate the similar results, though estimates from NPCPM-FIGRACH models are relatively smaller. However, compared to the estimates of $d$ from original FIGARCH model, the differences are fairly small.

For the 3S-FIGARCH model, it achieves the smallest AIC and BIC and leads to the smallest estimates of $d$ in all cases. In terms of AIC and BIC, the improvements of 3S-FIGARCH models over NPCPM-FIGARCH mod-

\footnotetext{
${ }^{6}$ We also fit 3S-FIGARCH models with different threshold probabilities to robust our estimates. The results are available upon request.

${ }^{7}$ To make the models parsimonious, we set both $K$ and $R$ to be 1 for A-FIGARCH and TV-FIGARCH models, respectively, in all cases.
} 
els are much greater than those of NPCPM-FIGARCH models over A-FIGARCH or TV-FIGARCH models. For example, for Nasdaq, the difference between BIC of 3S-FIGARCH and NPCPM-FIGARCH models with Student-t distribution is more than 300. The difference between BIC of NPCPM-FIGARCH models and AFIGARCH or TV-FIGARCH models, all with Student-t distribution, is less than 100. Besides, 3S-FIGARCH framework (number of variable is 8) is more parsimonious compared to NPCPM-FIGARCH framework (number of variable is 16 for Nasdaq). As a result, this demonstrates that using the information of $P\left(s_{t}=1 \mid \theta, \Omega_{T}\right)$ to identify the economic states can lead to much better model performance. Finally, 3S-FIGARCH model produces the smallest estimate of $d$. Compared to the estimate from the original FIGARCH model, the difference can be up to more than $30 \%$. This further confirms that using the information of $P\left(s_{t}=1 \mid \theta, \Omega_{T}\right)$ to identify the economic states is much more effective and reliable to estimate long memory parameter than the existing approaches.

\section{Conclusion}

This paper proposes a two-stage 3S-FIGARCH model as a new approach to detect the structural breaks and incorporate it into the FIGARCH framework. By using the hourly returns of stock indexes Nasdaq and ASX from $1 / 1 / 2001$ to $31 / 12 / 2012$, we demonstrate that 3S-FIGARCH model outperform the original and existing FIGARCH frameworks which incorporate the structural breaks by modelling intercept in the conditional variance equation via certain time dependent function (A-FIGARCH and TV-FIGARCH models). This conclusion still holds when compared to NPCPM-FIGARCH framework which also detects change points and model FIGARCH process in separate stages. In addition, Diebold and Inoue (2001) argue that long memory can be caused by structural breaks and is easily confused with it. Therefore, the fact that the estimated $d$ from $3 \mathrm{~S}$ FIGARCH framework is smaller than those from other FIGARCH models further confirms that our proposed approach can lead to more effective and reliable estimate of long memory parameter. Thus, our 3S-FIGARCH framework could be a widely useful tool for modelling long memory persistence of high-frequency financial volatility in other contexts.

\section{REFERENCES}

Baillie, R. T., T. Bollerslev, and H. O. Mikkelsen (1996). Fractionally integrated generalized autoregressive conditional heteroskedasticity. Journal of Econometrics 74(1), 3 - 30.

Baillie, R. T. and C. Morana (2009). Modelling long memory and structural breaks in conditional variances: An adaptive figarch approach. Journal of Economic Dynamics and Control 33(8), 1577-1592.

Belkhouja, M. and M. Boutahary (2011). Modeling volatility with time-varying figarch models. Economic Modelling 28(3), 1106-1116.

Bollerslev, T. (1986). Generalized autoregressive conditional heteroskedasticity. Journal of Econometrics 31(3), 307-327.

Diebold, F. X. and A. Inoue (2001). Long memory and regime switching. Journal of Econometrics 105(1), $131-159$.

Franses, P. and D. van Dijk (1996). Forecasting stock volatility using (non-linear) garch models. Journal of Forecasting 15, 229-235.

Haas, M. and M. S. Paolella (2012). Mixture and Regime-Switching GARCH Models. United Kingdom: Wiley.

Hamilton, J. D. (1989). A new approach to the economic analysis of nonstationary time series and the business cycle. Econometrica 57(2), 357-384.

Ho, K.-Y., Y. Shi, and Z. Zhang (2013). How does news sentiment impact asset volatility? evidence from long memory and regime-switching approaches. North American Journal of Economics and Finance.

Ross, G. J. (2013). Modelling financial volatility in the presence of abrupt changes. Physica A: Statistical Mechanics and its Applications 392(2), 350-360.

Susmel, R. and R. F. Engle (1994). Hourly volatility spillovers between international equity markets. Journal of International Money and Finance 13(1), 3-25.

Wilfling, B. (2009). Volatility regime-switching in european exchange rates prior to monetary unification. Journal of International Money and Finance 28(2), 240-270. 